REVIEW ARTICLE

\title{
COVID-19 Pathological Perspective: A Review
}

\author{
Anandraj Vaithy ${ }^{1}$, Venkat Raghavan ${ }^{2}$, Sowmya Srinivasan ${ }^{3}$, Umadevi Ramachandra $^{4}$
}

\begin{abstract}
COVID-19, being a dreadly viral infection, has put the entire globe in a pandemic situation in the present scenario. The RNA virus was first reported in later 2019; various diagnostic modalities are applied including polymerase chain reaction (PCR) since PCR detects RNA viruses and their strain. Though many theories had been proposed and accepted worldwide, the path and sequelae of COVID-19 is debatable especially after the release of the autopsy analysis conducted at Italy, the results of which turned out to be a revolutionary tool in managing COVID-19 patients. The coronavirus could be classified based on its characteristics into many types such as O, A2, A2a, A3, B, B1, totally numbering to 11 strains. It has been proved by research studies including an Indian institute's study that "A2a" strain is the most predominant and virulent strain. The basic pathogenic molecular mechanism of SARS-Cov2 is by entering the lung system and cells and disrupting the 1- $\beta$ chain of hemoglobin and extracting out the porphyrin rings and dissociating the iron content from it, thereby releasing iron into the circulation. The increased $\mathrm{Hb}$ production eventually leads to increase in the blood viscosity level with recurrent and diffuse micro- and macrocirculatory thrombosis in the form of blood clot, the major underlying reason substantiating the elevated levels of D-dimer among the infected patients and thus explaining the cause of sudden deterioration and death. COVID-19 management warrants a multidimensional approach in management, and judicious laboratory diagnosis plays a vital role in picking up the infected cases. Scientific reviews throws light on the primary underlying pathophysiology, thereby redirecting the mode of approach and subsequent management and proclaiming that the grave condition is associated with disseminated intravascular coagulation with microthrombi warranting rationale treatment with anti-inflammatory and anti-coagulant drugs rather than empirical and irrational usage of mechanical ventilators.
\end{abstract}

Keywords: Anticoagulants, Autopsy, COVID-19, Disseminated intravascular coagulation.

SBV Journal of Basic, Clinical and Applied Health Science (2020): 10.5005/jp-journals-10082-02247

\section{INTRODUCTION}

The novel coronavirus disease 2019, termed as COVID-19, was first said to be reported in Wuhan domicile, China, in the later part of the year 2019 (reportedly from December 2019); it had turned out to be pandemic and strongly associated with sequelae of adult respiratory distress syndrome (ARDS). ${ }^{1,2}$ The dreadly virus is known to infect all age groups from newborn to old age with worse outcomes among elderly. 2,3 The severity of the virus depends on the strain, and it has been identified that the virus prevails in several mutated forms among which 10 forms had been proved to scientifically exist. Among all the prevailing forms, the strain "A2a" had evolved as a dominant strain and almost replaced all forms occurring worldwide. It had been proved by several studies including an Indian study conducted by Indian Institutes of Research that "A2a" strain is the predominant virulent strain. ${ }^{3,4}$

An epidemiological study by veteran researchers from the National Institute of Biomedical Genomics (NIBG) located in Kaylan region of West Bengal proved that the novel coronavirus with the A2a mutation is highly potent in invading human lung cells in substantial numbers. ${ }^{2,3}$

Studies done by Paratha Mujumdar from Indian Council for Medical Research (ICMR) and other nations showed that the severity of the systemic immune response in the form of inflammation as a reaction to COVID-19 species had features reminiscent of cytokines termed under the broad category "macrophage activation syndrome." ${ }^{2-4}$ Few researchers also call the term "secondary hemophagocytic lymphocytosis." ${ }^{5-7}$ The present pandemic scenario varies from the previous SARS-COVID virus that ended up with 800 mortalities among 8,000 infected peoples, which occurred a decade back. The pathophysiology involves the similar mode of transmission in entering the lungs, however not as virulent as A2a. All the analytical studies carried out are very crucial in terms \begin{tabular}{l}
\hline${ }^{1-4}$ Department of Pathology, Mahatma Gandhi Medical College and \\
Research Institute, Sri Balaji Vidyapeeth, Puducherry, India
\end{tabular}

Corresponding Author: Anandraj Vaithy, Department of Pathology, Mahatma Gandhi Medical College and Research Institute, Sri Balaji Vidyapeeth, Puducherry, India, Phone: +91 9486142544, e-mail: kanandrv@gmail.com

How to cite this article: Vaithy A, Raghavan V, Srinivasan S, Ramachandra U. COVID-19 Pathological Perspective: A Review. J Basic Clin Appl Health Sci 2020;3(2):70-74.

Source of support: Nil

Conflict of interest: None

of clinical outcomes since they provide a platform for vaccine manufacturers with a specific target. ${ }^{8,9}$

The initial diagnosis was that the coronavirus has evolved into newer strains during its transmission within China and then onward the remainder of the planet. The virus is classified based on its specifications into many types such as $\mathrm{O}, \mathrm{A} 2, \mathrm{~A} 2 \mathrm{a}, \mathrm{A} 3, \mathrm{~B}, \mathrm{~B} 1$, etc. Whereas the strain $O$ is the "ancestral type" and proved to be evolved in Wuhan, as studied by researchers. ${ }^{9}$

To live and survive, a virus should propagate and disseminate by infecting other animals. With this basis, a mutation usually disables an infective form of virus from transmitting itself. In exceptions, some mutations enable the virus to transmit and propagate more rapidly to cause community spread. The SARSCoV2 is observed to be following such transmission as documented by ICMR scientists. ${ }^{10}$ Coronavirus infection, also known as COVID, after gaining contact is known to begin infectious sequelae in the patient's throat, thereby seeking entry into lungs and replication, causing breathing difficulties and eventually respiratory distress. Scientists had observed that the A2a mutation made a significant

(c) The Author(s). 2020 Open Access This article is distributed under the terms of the Creative Commons Attribution 4.0 International License (https://creativecommons. org/licenses/by-nc/4.0/), which permits unrestricted use, distribution, and non-commercial reproduction in any medium, provided you give appropriate credit to the original author(s) and the source, provide a link to the Creative Commons license, and indicate if changes were made. The Creative Commons Public Domain Dedication waiver (http://creativecommons.org/publicdomain/zero/1.0/) applies to the data made available in this article, unless otherwise stated. 
alteration in the contents of the spike protein (the major protein molecule present on the surface of the cell membrane) of the coronavirus, allowing it to gain more access for binding easily with the surface protein present in the lung cell. ${ }^{1}$ Thus, COVID-19 has emerged as a deadly pandemic owing to the A2a type's ability to transmit easily. ${ }^{11,12}$ The clinical demographics of the A2a mutant strain is depicted in Figure 1.

\section{Viral Characteristics}

COVID-19 is a RNA virus; the weight of a virus is measured to be 0.85 actograms or $0.85 \times 10^{-18} \mathrm{~g}$, or about one millionth of a trillion grams. Seventy billion viruses that will make an individual sick attract about $0.0000005 \mathrm{~g}^{13}$ Since the entire number of cases worldwide is now over 2 million, the entire weight of the rogue viruses that descend the planet comes about $1 \mathrm{~g}$. The entire mankind is on its knees with $1 \mathrm{~g}$ of virus. It has unique way of invading the pneumocytes causing morbidities as pictorially portrayed in Figure 2.

\section{Scientific Analysis-Breakthrough}

The COVID-19 pandemic has resulted in a high incidence of morbidity and mortality throughout the world since the outbreak of H1N1 influenza pandemic in 1918-which is reported to be the deadliest pandemic to have occurred for a century. The causative virus, SARS-CoV-2, is a newly described genus identified to be belonging to the family Coronaviridae and genus $\beta$ coronavirus. ${ }^{14}$

Several studies and pilot analysis on molecular parameters done by Indian scientists had come out with substantial evidences and explanation toward the mechanism and pathways involved in the morbidity sequelae of COVID-19. 2,15,16 The postulated analysis by Indian and worldwide researchers had scientific consensus as follows.

COVID-19 infection might result in pneumonia as such either in the typical form or atypical or classical respiratory distress. It seems like clinicians and pulmonologist are dealing with a presumed empirical disease rather than thinking on the hemostatic sequelae with concept of microthrombi. ${ }^{2}$

\section{Covid-19 and Pulmonary System}

Macrophage activation syndrome (MAS) is an event triggered by cytokine storm usually treated with anticytokine therapies. Macrophage activation syndrome-induced pulmonary immunopathology is a characteristic of pneumonia due to COVID19 with manifestation of hemophagocytic lymphocytosis as a cardinal presentation. Extensive infiltration of the pulmonary system by macrophages and other immune system-related cells subsequently leads to diffuse alveolar damage commonly termed as COVID-19-induced pneumonia. It can be viewed radiographically as well in the form of consolidated patches as shown in Figure 3.

The major virulent step of SARS-Cov2 is to invade the hemoglobin especially beta chain group and thereby extracting porphyrin groups leading to dissociation of the iron form and leading to free release of the iron form into the circulatory system. As a sequelae, hemoglobin loses its nature of binding capacity to oxygen molecules leading to systemic hypoxia. ${ }^{3}$ This hypoxia triggers several cascade of events leading to alteration in hemostasis, thereby causing multiorgan failure syndrome as depicted in Figure 4.

The iron content dissociated from heme gets released into circulation and it is inherently harmful due to its oxidative stressinduced cell injury causing lung distress. Free iron toxicity causes diffuse inflammation particularly alveolar macrophages present in the lungs, which could be evidenced radiographically. ${ }^{4}$ The hemostatic system reacts to compensate this loss of heme by increasing the rate of synthesis of hemoglobin $(\mathrm{Hb})$, thus explaining the reason for encountering high hemoglobin value among the COVID-19 patients. Several compensatory mechanisms come in force to act upon such ferritin, acute-phase reactants, reactive monocytosis, and increased blood viscosity, leading to formation of microthrombi in circulation as evidenced by elevated $D$-dimer levels among the infected patients. The presence of disseminated microthrombi is also proved by autopsy studies conducted in European countries like Italy among respiratory distress victims due to COVID-19. ${ }^{5}$

A panel of immune factors contributes to formation of pulmonary intravascular coagulopathy as mentioned below: ${ }^{6}$

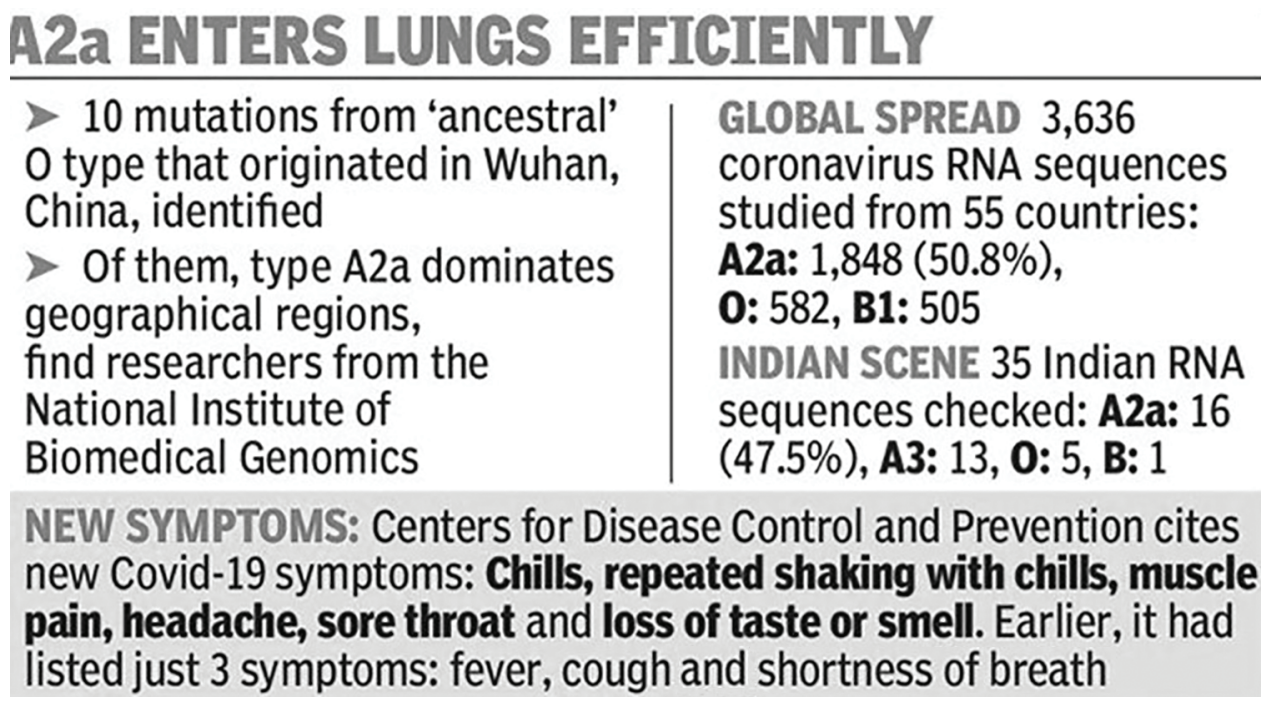

Fig. 1: Clinicosocial demographics of A2a strain of COVID-19 


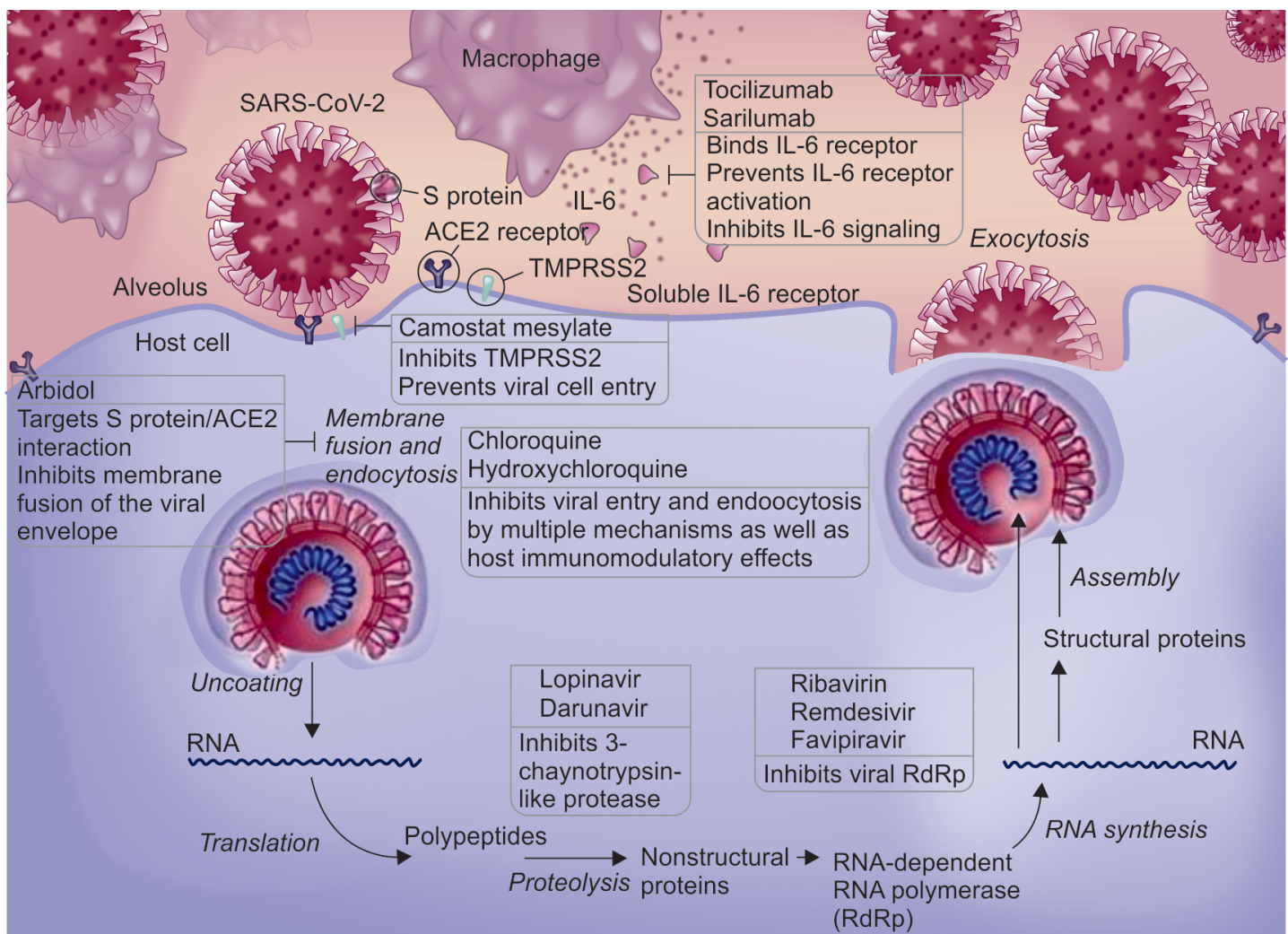

Fig. 2: Pathophysiology of virus invading pneumocytes and action of management drugs. PC: Lancet DOI: 10.1016/S2665-9913(20)30121-1

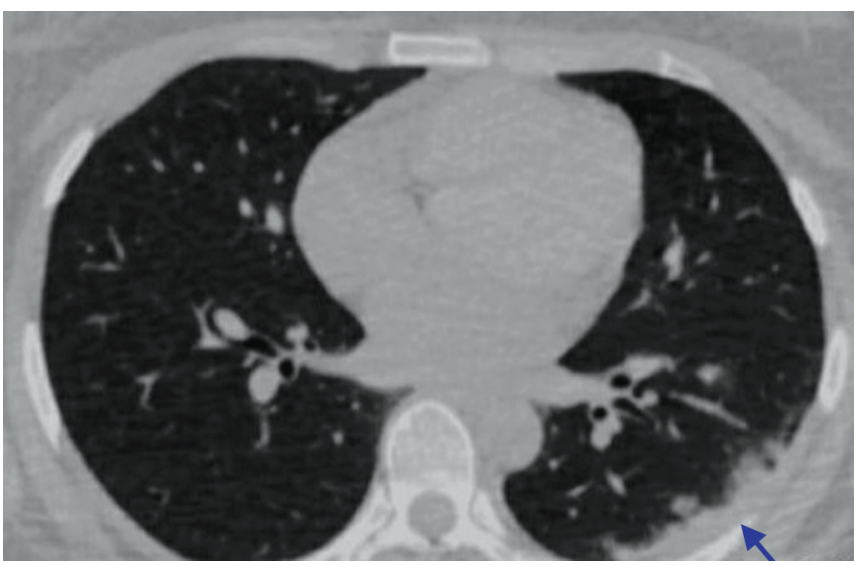

Fig. 3: Chest radiographs showing peripheral consolidation (arrow)

- Diffuse pattern of alveolar collapse and systemic inflammation triggering chemical mediators

- Interstitial inflammation

- Macrophage activation mechanism of the pulmonary system

- Disruption in the innate immune responses of the pulmonary system

- Senile coagulation cascade changes

- Mechanism of triggering immunothrombosis

Thus, the theory substantially explained the reason for high surge in the mortality rate, proving that mechanical ventilation is ineffective in treatment and indeed few authors propose that mechanical ventilation could actually be harmful causing more lung damage rather than treating the disease. ${ }^{7,8}$
In Italy, observations from 50 autopsies performed on patients who succumbed to COVID-19 revealed that the condition is not pneumonia per se as assumed, since the virus does not only destroy the pneumocytes (types I and II) but also stimulate an inflammatory cascade leading to endothelial vascular thrombosis with subsequent diffuse thrombosis of the lung and it produces an attack or stroke and subsequently other thrombotic diseases. ${ }^{9}$ In fact, the protocols that are widely being followed using antiviral therapies have become questionable and enlightened to concentrate on the inflammatory and anti-clotting drugs. Thus, the lung damage caused by COVID-19 is entirely different from that of bronchopneumonia as pictorially depicted in Figure 5.

The proposed multidimensional therapies must be started immediately, even at initial stage, where the treatment responds alright to the patients, thus proving the efficacy of anti-inflammatory drugs and anti-coagulants with antibiotics. ${ }^{10}$

An Italian anatomical research pathologist reported a total of 50 autopsies conducted at Pergamo hospital and 20 autopsies in Milan. The clinical outcome is decided by the extent of disseminated intravascular coagulation activated by the virus; hence, respiratory disease doesn't have any role and it seem to be an enormous diagnostic error. Thus, in retrospect, we have to redirect the diagnostic modalities and these chest radiographs that were assumed to be interstitial pneumonia could actually be fully consistent with a disseminated interstitial coagulation. ${ }^{11,12}$

Patients were referred to intensive care units with indication for thrombus and generalized venous embolism, generally lupus as underlying indication, and thus intubations and resuscitations would be useless if thromboembolism is left untreated. Ventilating a lung where blood does not reach is less helpful. ${ }^{12,13}$ The authors also revealed that 9 out of 10 patients infected with COVID-19 die 

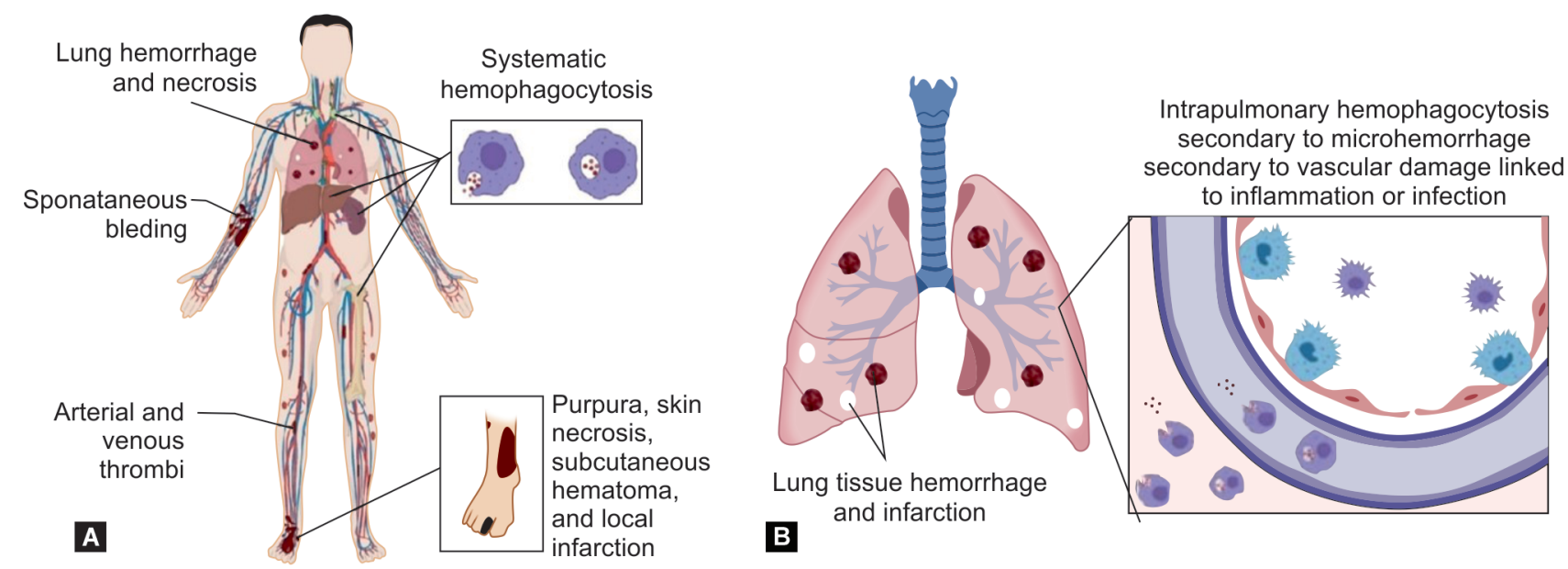

Figs 4A and B: Early macrophage activation syndrome vs early COVID-19. (A) Secondary hemophagocytic lymphohistiocytosis or macrophage activation syndrome is associated with organomegaly, thrombocytopenia, hemophagocytosis, and disseminated intravascular coagulation; (B) Pulmonary involvement without generalized lymphoid organ hyperplasia is typical of COVID-19 pneumonia. Hemophagocytosis. PC: Lancet Rheumatology, 2020

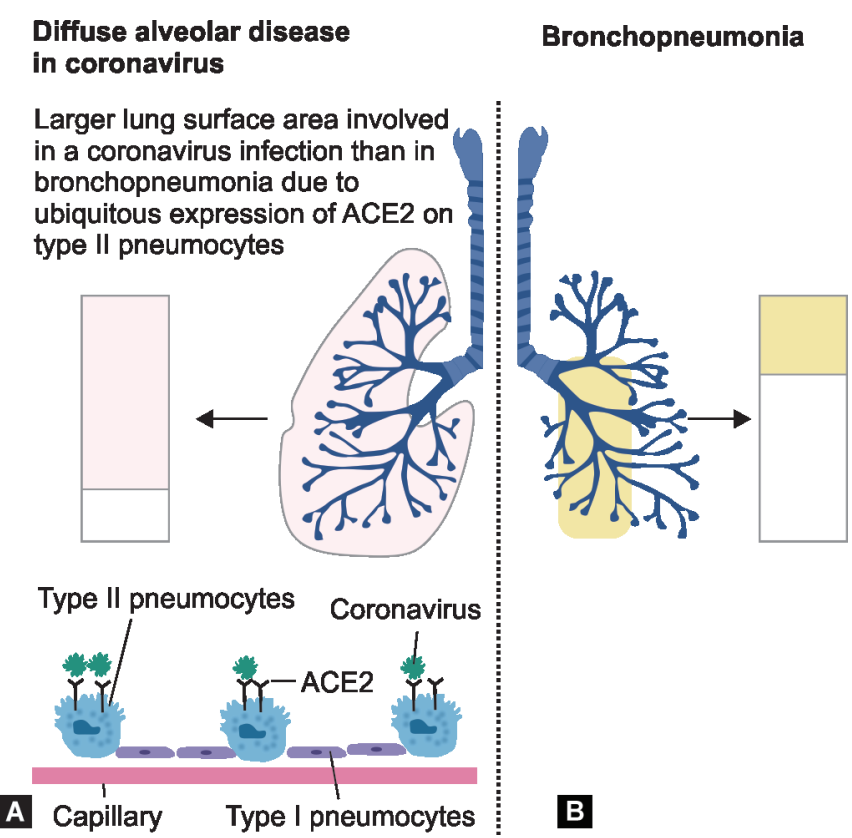

Figs 5A and B: Extent of alveolar lung surface involved in COVID-19 and bronchopneumonia; (A) COVID-19 gain access to the lungs via the ACE2 receptor that is expressed most abundantly on a subpopulation of type II pneumocytes; (B) Segmental bronchopneumonia typically has a different lung distribution, with prominent bronchial tree involvement. Pc: Lancet J Rheumat

owing to the problem in cardiovascular system not pulmonology causes. ${ }^{14,15}$ It is the presence of venous microthrombosis and not pneumonia that determines mortality as assumed. ${ }^{16-18}$

\section{Conclusion}

The present review emphasizes that a multidimensional approach is essential in managing COVID-19 pandemic. Laboratory diagnosis plays a vital role in picking up the infected cases. Scientific reviews throw light on the primary underlying pathophysiology, thereby redirecting the mode of approach and subsequent management and proclaiming that the condition is associated with disseminated intravascular coagulation with microthrombi warranting rationale treatment with anti-inflammatory and anti-coagulant drugs rather empirical and irrational usage of mechanical ventilators. Exploration is needed to prove that COVID-19 is not just a pneumonia but also a venous microthrombi condition and coagulopathy-associated disorder.

\section{References}

1. Team NCPERE. Vital surveillances: the epidemiological characteristics of an outbreak of 2019 novel coronavirus diseases (COVID-19) - China. Team NCPERE. China CDC Weekly 2020; vol. 28. pp. 113-122.

2. Indian Council for Medical Research. COVID-19 testing. [Online] [Cited: March 28, 2020.] https://www.mohfw.gov.in/pdf/Advisoryontheuse ofHydroxychloroquinasprophylaxisforSARSCoV2infection.pdf.

3. EMR Division, Ministry of Health and Family Welfare, Government of India. Guidelines on Clinical Management of COVID-19 infection. [Online] https://www.mohfw.gov.in/pdf/Guidelines on Clinical Management of COVID 19 12020.pdf.

4. Wu Y, Guo C, Tang L, Wang C, Luo F, Yu X, et al. Prolonged presence of SARS-CoV-2 viral RNA in fecal samples. Lancet Gastroenterol Hepatol 2020;5(5):434-435. DOI: 10.1016/S2468-1253(20)30083-2.

5. Tang B, Xia F, Tang S, Bragazzi NL, Li Q, Sun X, et al. The effectiveness of quarantine and isolation determine the trend of the COVID-19 epidemics in the final phase of the current outbreak in China. Int J Infect Dis 2020;95:288-293. DOI: 10.1016/j.ijid.2020.03.018.

6. Tang N, Li D, Wang X, Sun Z. Abnormal coagulation parameters are associated with poor prognosis in patients with novel coronavirus pneumonia. J Thromb Haemost 2020;18(4):844-847. DOI: 10.1111/ jth.14768.

7. McGonagle D, Sharif K, Regan A, Bridgewood C. The role of cytokines including interleukin- 6 in COVID-19 induced pneumonia and macrophage activation syndrome-like disease. Autoimmun Rev 2020;19(6):102537. DOI: 10.1016/j.autrev.2020.102537.

8. George MR. Hemophagocytic lymphohistiocytosis: review of etiologies and management. J Blood Med 2014;5:69-86. DOI: 10.2147/ JBM.S46255.

9. Shoenfeld Y. Corona (COVID-19) time musings: our involvement in COVID-19 pathogenesis, diagnosis, treatment and vaccine planning. Autoimmun Rev 2020;19(6):102538. DOI: 10.1016/j.autrev.2020. 102538.

10. Hsueh PR, Chen PJ, Hsiao CH, Zhou J, Dong X, Yin H. Patient data, early SARS epidemic, Taiwan. Emerg Infect Dis 2004;10(3):489-493. DOI: 10.3201/eid1003.030571. 
11. Nicholls JM, Poon LL, Lee KC. Lung pathology of fatal severe acute respiratory syndrome. Lancet 2003;361(9371):1773-1778. DOI: 10.1016/S0140-6736(03)13413-7.

12. Seguin A, Galicier L, Boutboul D, Lemiale V, Azoulay E. Pulmonary involvement in patients with hemophagocytic lymphohistiocytosis. Chest 2016;149(5):1294-1301. DOI: 10.1016/j.chest.2015.11.004.

13. Franks TJ, Chong PY, Chui P, Ren J, Zhao Y, Hu L. Lung pathology of severe acute respiratory syndrome (SARS): a study of 8 autopsy cases from Singapore. Hum Pathol 2008;34(8):743-748. DOI: 10.1016/ S0046-8177(03)00367-8.

14. Yao XH, Li TY, He ZC, Wen R, Sun P, Xing QS. A pathological report of three COVID-19 cases by minimally invasive autopsies. Zhonghua Bing Li Xue Za Zhi 2020;49(5):411-417. DOI: 10.3760/cma. j.cn112151-20200312-00193.

15. Tian S, Hu W, Niu L, Liu H, Xu H, Xiao SY. Pulmonary pathology of early-phase 2019 novel coronavirus (COVID-19) pneumonia in two patients with lung cancer. J Thorac Oncol 2020;15(5):700-704. DOI: 10.1016/j.jtho.2020.02.010.

16. Smeeth $L$, Thomas $S L$, Hall AJ, Hubbard R, Farrington P, Vallance P. Risk of myocardial infarction and stroke after acute infection or vaccination. N Engl J Med 2004;351(25):2611-2618. DOI: 10.1056/ NEJMoa041747.

17. Corrales-Medina VF, Musher DM, Wells GA, Chirinos JA, Chen L, Fine MJ. Cardiac complications in patients with community acquired pneumonia: incidence, timing, risk factors, and association with short-term mortality. Circulation 2012;125(6):773-781. DOI: 10.1161/ CIRCULATIONAHA.111.040766.

18. Hwang DM, Chamberlain DW, Poutanen SM, Low DE, Asa SL, Butany J. Pulmonary pathology of severe acute respiratory syndrome in Toronto. Mod Pathol 2005;18(1):1-10. DOI: 10.1038/ modpathol.3800247. 